\title{
Diferentes estratégias para determinação da matriz hessiana e do parâmetro de barreira e o problema de fluxo de potência ótimo
}

\author{
Gabriela F. Bregadioli \\ Programa de Pós-Graduação em Engenharia Elétrica, UNESP-Univ. Estadual Paulista \\ Av. Eng. Luiz Edmundo C. Coube, 14-01, 17033-360, Bauru, SP \\ E-mail: gaby_fb.dc@hotmail.com \\ Edméa C. Baptista \\ Departamento de Matemática, UNESP-Univ. Estadual Paulista \\ Av. Eng. Luiz Edmundo C. Coube, 14-01, 17033-360, Bauru, SP \\ E-mail: baptista@fc.unesp.br \\ Leonardo Nepomuceno \\ Departamento de Engenharia Elétrica, UNESP-Univ. Estadual Paulista \\ Av. Eng. Luiz Edmundo C. Coube, 14-01, 17033-360, Bauru, SP \\ E-mail: leo@feb.unesp.br
}

\section{Introdução}

\section{RESUMO}

A consolidação de técnicas matemáticas para resolução de problemas de grande porte, o surgimento de novos e potentes recursos computacionais, a um custo relativamente baixo, e o aumento no interesse econômico na operação dos sistemas de energia vem impulsionando a utilização dos problemas de fluxo de potência ótimo (FPO) como ferramenta para a análise da operação dos sistemas de potência. A primeira formulação do problema de FPO é atribuída a Carpentier [3] na década de 60. O principal objetivo do problema de FPO é determinar o estado de operação ótimo de um sistema de potência e ele pode ser modelado como um problema de programação não linear (PNL). Em geral, esses modelos são não lineares, não convexos e de grande porte, tornando-os difíceis de serem resolvidos. Com o intuito de amenizar esta dificuldade, muitos pacotes de algoritmos de otimização foram desenvolvidos e podem ser aplicados na resolução destes tipos de problemas [1]. Destacamos aqui o pacote de otimização denominado Knitro [5]. A robustez, eficiência e facilidade de manipulação deste pacote de otimização torna-o uma ferramenta potente e este, pode ser utilizado em redes de transmissão com um enorme número de barras.

O Knitro tem como base um método de pontos interiores e um método de conjunto ativo, e apresenta quatro diferentes estratégias para a determinação da matriz hessiana: (a) Hessiana Exata; (b) Quasi-Newton BFGS; (c) Quasi-Newton SR1; e (d) Quasi-Newton BFGS com memória limitada; bem como seis estratégias para a atualização do parâmetro de barreira: (i) o parâmetro de barreira é decrescido monotonamente; (ii) o parâmetro de barreira é calculado por uma regra adaptativa com base na diferença de complementaridade; (iii) é realizada uma investigação sobre o passo (afimescala) para determinar dinamicamente o valor do parâmetro barreira a cada iteração; (iv) é utilizada uma regra Mehrotra tipo preditor-corretor para determinar o parâmetro de barreira levando em conta o passo corretor; (v) é utilizada uma regra Mehrotra tipo preditor-corretor para determinar o parâmetro de barreira sem considerar o passo corretor; e (vi) uma função mérito é minimizada em cada iteração para determinar o parâmetro de barreira.

Neste sentido, propomos neste trabalho uma análise das estratégias para a determinação da matriz hessiana, juntamente com as regras de atualização do parâmetro de barreira, na solução problema de FPO, utilizando o pacote de otimização Knitro.

\section{O Problema de FPO}

O problema de FPO, matematicamente, pode ser modelado como:

$$
\begin{array}{lll}
\min & f(x) & \\
\text { s.a.: } & g_{i}(x)=0 & i=1,2, \ldots, m<n \\
& h_{j}(x) \leq 0 & j=1,2, \ldots, p \\
& x_{\min } \leq x \leq x_{\max } &
\end{array}
$$


em que $\mathrm{x}^{\mathrm{T}}=(\mathrm{V}, \theta) \in \mathbb{R}^{\mathrm{n}}$ é o vetor das variáveis de estado e de controle; $\mathrm{f}(\mathrm{x})$ é a função objetivo que, neste trabalho, representa as perdas de potência ativa nas linhas de transmissão; $\mathrm{g}_{\mathrm{i}}(\mathrm{x})$ correspondem às equações do fluxo de potência; $h_{j}(x)$ correspondem às inequações funcionais da rede elétrica; $\mathrm{x}_{\min } \mathrm{e}$ $\mathrm{x}_{\max }$ são, repectivamente, os limites inferiores e superiores das variáveis de estado e controle.

\section{Testes Numéricos}

O sistema elétrico de potência usado para os testes numéricos foi IEEE 14 barras e tem as seguintes características: 1 barra de geração (slack); 4 barras de controle reativo; 9 barras de carga; 20 linhas de transmissão. Os dados deste sistema foram obtidos na página http://www.ee.washington.edu/research/pstca/, os taps dos transformadores foram considerados fixos e os limites mínimos e máximos das magnitudes de tensão das barras do sistema foram, respectivamente, $V_{\min }=0,95$ p.u. e $V_{\max }=1,1$ p.u. Um programa em scilab-5.4.1 foi desenvolvido para a leitura dos dados.

A formulação matemática do problema de FPO IEEE 14 barras contempla 27 variáveis, 23 restrições de igualdade e 38 restrições de desigualdade.

\section{Tabela 1. Convergência FPO 14 barras em número de iterações}

(Parâmetro de Barreira)

\begin{tabular}{|c|c|c|c|c|c|c|}
\hline & (i) & (ii) & (iii) & (iv) & (v) & (vi) \\
\hline \multirow{4}{*}{ 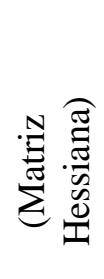 } & 12 & 11 & 8 & 7 & 7 & 8 \\
\hline & 23 & 12 & 10 & 9 & 8 & 11 \\
\hline & 20 & 12 & 10 & 9 & 9 & 10 \\
\hline & 29 & 17 & 14 & 18 & 14 & 17 \\
\hline
\end{tabular}

Na tabela 1 observamos que, para a matriz hessiana exata (a), as estratégias (iv) e (v) foram as mais eficientes. Já para todas as estratégias que utilizam as matrizes hessianas de maneira aproximada (b), (c) e (d), destacamos a estratégia (v). Observamos que ocorreu um empate no caso (d) entre as estratégias (iii) e (v) e que dentre as estratégias aproximadas a (b) juntamente com a (v) é a que resultou no menor número de iterações. Estudos com sistemas maiores estão sendo realizados.

Palavras-chave: Método de Pontos Interiores, Fluxo de Potência Ótimo, Hessiana ,Knitro.

\section{Referências}

[1]M. F. Bedriñana, M. J. Rider, C. A. Castro, Ill-conditioned Optimal Power Flow Solutions and Performance of Non-Linear Programming Solvers, em "IEEE Bucharest Power Tech Conference", pp. 01-07, Bucharest, Romania, 2009.

[2] R. H. Byrd, J. Nocedal, R. A. Waltz, KNITRO: An integrated package for nonlinear optimization, Large-Scale Nonlinear Optimization. New York, Springer Verlag, p. 3, 2006.

[3] J. L. Carpentier, Contribution a L'etude du Dispatching Economique. Bull-Soc. Fr Elec., Ser. B3, $431-447,1962$.

[4]E. C. Ferreira, "Uma investigação da influência do parâmetro de barreira na solução do problema de fluxo de potência ótimo", Dissertação de Mestrado, Faculdade de Engenharia de Bauru, Universidade Estadual Paulista, Bauru, 2013.

[5]R. A. Waltz, T. D. Platenga, "Knitro User's Manual. Version 9.0", Technical Report, Ziena Optimization, Evanston, Il, Usa, 2013. 